\title{
To Study Outcome of Concurrent Chemo-Radiation with Cisplatin in Advanced Case of Oral Malignancy
}

\author{
Aman Agarwal \\ Department of Surgery, Krishna Institute of Medical Sciences, Malkapur, Karad, Dist. Satara, Maharastra-415110, India
}

\begin{abstract}
Cancer is a major cause of death in the world Oral cancer has posed a challenge to us with its incidence as high as around $20 \%$ of total body cancers $7 \%$ of all cancer deaths in males and $4 \%$ in females have been reported to be due to oral malignancy recent meta-analyses indicate that concurrent chemoradiation yields an $8 \%$ absolute increase in overall survival at 5 years compared to radiotherapy alone. The aim is to study the outcome of concurrent chemo-radiation with cisplatin in advanced case of oral malignancy. Methods: Between Nov. 2013 and June 2015, 50 patients in good condition ranging between 50 to 100 on karnofsky scale with advanced oral cancer treated with combination of chemotherapy \& Radiotherapy. The present study included 50 patients of stage III and stage IV oral cancer 35 were male and 15 were females their age varied between 30 yrs to 80 yrs. • General physical examination - Systemic examination - Local examination• Complete blood investigation (Haemogram, RFTs, LFTs)・ Biopsy from the primary tumour and FNAC from metastatic L.Node. Dental evaluation and chest X-ray and X-ray mandible. $\bullet$ CT scan head and neck Results: The commonest age group was the 4th, 5th and 6th decade. The average age is 52. The male to female ratio is 2.3:1.These clearly showed preponderance of male. Tobacco chewing is the most common habit in male patient and Mishri application in female patients. Exophytic lesion were high 34/50 cases and commonest in Tongue 46\%. Most common site is Tongue 46\%. Histopathologicaly well differentiated squamous cell carcinoma is commonest i.e 24 cases $(48 \%)$ followed by moderately diff. squamous cell carcinoma. incidence of advanced oral malignancy more in Lower (Poor) socioeconomic class than higher + middle class ie. 4.5:1. 72\% cases were having poor oral hygiene. It can be seen in the above table response rate in the case of stage III in $100 \%$ \& stage IV $90 \%$. Well differentiated squamous cell carcinoma shows $100 \%$ response to combined therapy where as poor differentiated shows $100 \%$ of no response. Conclusion: This study included fifty patients of Stage III and Stage IV oral cavity cancer. Thirty five were male and fifteen were female. Average age of disease was 52 years a commonest decade being the 5th decade. clear male preponderance Poor oral hygiene was observed in $72 \%$ majority of cases from lower socioeconomic class i.e. $82 \%$ The most common habit in male is chewing tobacco and in female is tobacco mishri application Most of the cases presented in the study between 3 to 4 months i.e. $54 \%$ cases. commonest site of malignancy was tongue followed by buccal mucosa. $48 \%$ cases were well-differentiated squamous cell carcinoma. 25 of them had complete remission and 21 patients had partial remission. 4 patients delivered the no response. In conclusion combination of cisplatin - and radiotherapy given as once weekly and RT for 33 Fr 5 times a week found to be effective, economical and was better tolerated by the patients.
\end{abstract}

Keywords: Concurrent, Radiotherapy, Cisplatin, Karnofsky scale, well diff.squamous cell carcinoma, complete remission, oral hygiene, tongue, Exophytic, partial remission, no response.

\section{Introduction}

Cancer is a major cause of death in the world. Cancer is often silent, hence diagnosis may not be possible until quite late but oral lesions can be readily inspected and be easily inspected, measured, biopsied and documented. Objective response to treatment can be easily appreciated.

Oral cancer has posed a challenge to us with its incidence as high as around $20 \%$ of total body cancers. It is one of the ten leading cancers in the World. In India it is one of the most common cancers and is an important public health problem. About 56000 new cases have been estimated to occur each year, which would lead to more than 100000 individuals suffering from the disease in the population in any given year, furthermore $7 \%$ of all cancer deaths in males and $4 \%$ in females have been reported to be due to oral malignancy ${ }^{1}$ Oral cancers are many a times preceded a premalignant lesion.

Failure of local control of disease is the usual cause of death in patients with advanced stage oral cavity tumours. If combination of chemotherapy and radiotherapy could substantially reduce the bulk of a tumour without prejudicing its blood supply, and hence its oxygenation.
Although primary modalities of definitive treatment with curative intent are radiation and surgery, these approaches fail to cure a sizeable percentage of patients. Consequently, use of combined chemotherapeutic agents and radiotherapy is being considered as another modality of treatment, especially as adjuvant therapy. The role of chemotherapy and raditherapy at present is palliative only in advanced oral cancer. In this preliminary clinical trial-conducted in our Unit, we have found that combination of radiotherapy and chemotherapy is effective as primary treatment modality in oral cavity cancer and palliation of symptoms.

\section{Aims}

To study outcome of concurrent chemo-radiation with cisplatin in advanced case of oral malignancy.

\section{Objectives}

- Treatment feasibility can be assessed.

- To know the result of this combination in advanced oral malignancy.

- Response of the combination depending on stage of disease and clinical type. 


\section{International Journal of Science and Research (IJSR) \\ ISSN (Online): 2319-7064}

Index Copernicus Value (2013): 6.14 | Impact Factor (2015): 6.391

\section{Materials and Methods}

Between Nov. 2013 and June 2015, 50 patients in good condition ranging between 50 to 100 on karnofsky scale with advanced oral cancer treated with combination of chemotherapy \& Radiotherapy. The present study included 50 patients of stage III and stage IV oral cancer 35 were male and 15 were females their age varied between 30 yrs to 80 yrs. All cases were classified according to the TNM system using American Joint Committee of Cancer (AJCC) method.

\section{Inclusion Criterias}

1)Patients with histologically proven squamous cell carcinoma of oral cavity.

2)Normal hematological investigations.

3)Normal liver and renal function tests.

4)Normal chest x-ray

\section{Exclusion Criterias}

1) History of previous treatment of any modality including surgery radiotherapy and chemotherapy.

2) Patients with distant metastasis.

3) Patients with $\mathrm{Hb} \%(<10 \mathrm{gm} / \mathrm{dl})$

4) Patients with other co-morbid condition like Untreated TB, Uncontrolled diabetes, uncontrolled hypertension etc.

\section{Treatment Plan Scheme}

\section{Radiotherapy protocol:}

All patients will be treated by Cobalt-60 Teletherapy unit machine. The radiation dose delivered will be $66 \mathrm{~Gy}$ in 33Fr ( 2 Gy/Fr, 5 days in a week). The spinal cord will be shielded after 46 Gy in 23 Fr in some cases depending upon the initial treatment technique use.

\section{Chemotherapy Protocol:}

The drug cisplatin is used a single agent concurrently with radiotherapy. The dosage that will be used in $30 \mathrm{mg} / \mathrm{m}^{2}$ weekly for 6 cycles on same day of week. Premedication with dexamethasone, ranitidine and anti-emetics like granisetron will be given before cisplatin infusion. Adequate hydration consisting of dextrose normal saline added with KCL and mgso4 will be given before cisplatin infusion to body surface area will be added to normal saline and is given over 2 to 3 hrs IV infusion. Myelosuppression and renal toxicity will be evaluating by doing complete hemogram, blood urea and serum creatinine weekly.

\section{Follow-Up:}

The first follow up will be done at 4 weeks after the completion of treatment. During follow up the patients will be assessed for response of the primary. An attempt to follow up will be made each month till 6 months.

\section{Patient Selection}

1. Patient must have a histologically confirmed diagnosis of carcinoma of oral cavity

2. Patients of advanced oral cavity cancer stage III and stage IV were included only

3. Patient should not taken prior other therapy like, surgery, or radiotherapy.
4. The karnofsky scale should be $>50$

5. The Patient must have platelet count more than $1,00,000 / \mathrm{mm}^{3}$, white blood cell count more than $5000 / \mathrm{mm}^{3}$ and the haemoglobin more than $8 \mathrm{gm} \%$

6. Liver function test should be less than one standard deviation above normal.

7. Renal function must be within normal limit.

8. Patient should not have no other systemic disorder.

9. Patient should willingly give consent for undergoing the study and treatment.

\section{Dosage schedule}

Sandwich Therapy

1. Inj. Rantac $50 \mathrm{mg}$

(IV. bolus slow)

Inj. Dexa $8 \mathrm{mg}$

2. Inj. Kesol....(1) Amp in [500ml NS ]......(over 60 mints)

3. Inj. Pallostar $0.25 \mathrm{mg}$ in [500ml NS ].......(over 45 mints)

4. Inj. Cisplatin $-30 \mathrm{mg} / \mathrm{m}^{2}$ in [500ml NS ].......(over 90 mints)

5. IV . Mannitol $100 \mathrm{ml} . . . . \ldots \ldots \ldots \ldots \ldots . . . .(30 \mathrm{mints})$

6. Inj. MgSo4 (1) Amp in $500 \mathrm{ml} \mathrm{NS...........(over} 45$ mints)

7. IV. RL $500 \mathrm{ml}$ (over 45 mints)

Tab. Emeton 4mg TID (3 Day)

Tab. Pan 40 OD (3 Day)

Tab Domstal 10 mg BD (3 Day)

8. Patient is discharged on the same day after completing Cisplatin Therapy.

9. Radiotherapy is given 5 times a week for 33Fr of 66Gy.

10. After through clinico - Laboratory studies in term of platelet count, WBC count and haemoglobin gm\%, L.F.T., B.U.N. and S creatinine, X-Ray chest PAview X-Ray Mandible, biopsy of lesion, F.N.A.C. of node. The patient will be submitted for first dose in the first cycle.

11. The next cycle is to be repeated after one week on same day for 6 cycle.

12. Every time the clinical and laboratory work up will be done thoroughly and the next close is omitted at slightest suspicion of the toxicity.

Only objective response will be considered in assessing overall response, Response categories will includes:

1) Complete response: Complete disappearance of all clinical evidence of primary tumour and secondaries in lymph nodes.

2) Partial Response: A greater than $50 \%$ reduction in the product of two perpendicular dimensions or $50 \%$ reduction in squared diameter of primary site..

3) Any regression less than $100 \%$ but more than $50 \%$ will also be evaluated as partial response.

4) Any regression less than $50 \%$ but more than $25 \%$ will be evaluated as less than partial response (LPR).

5) Stable disease - No evidence of tumour progression established by caliper measurement or physical examination in prior sites at least for 60 days.

6) No response - Disease progressing inspite of therapy.

Observed toxicities and side effects:

1)Myelosuppression, Nausea, vomiting 


\section{International Journal of Science and Research (IJSR) \\ ISSN (Online): 2319-7064 \\ Index Copernicus Value (2013): 6.14 | Impact Factor (2015): 6.391}

2)Cirrhosis, pneumonitis, osteoporotic fractures, After methotrexate chemical pleuritis, therapy erythema, occular irritation anaphylaxis

3)Renal failure may occur during the course of treatment.

\section{Observation}

The study included 50 of advanced oral cavity malignancy which came to our hospital during two years Nov.2013 to June 2015.

Age distribution revealed that there were no cases below age 30 yrs and above 80 yrs.

Age distribution in advanced oral cavity cancer

\begin{tabular}{|c|c|c|c|c|}
\hline Age & Male & Female & Total & Percentage of Cases \\
\hline $30-40$ & 7 & 5 & 12 & $24 \%$ \\
\hline $41-50$ & 10 & 3 & 13 & $26 \%$ \\
\hline $51-60$ & 8 & 3 & 11 & $22 \%$ \\
\hline $61-70$ & 8 & 3 & 11 & $22 \%$ \\
\hline $71-80$ & 2 & 1 & 3 & $6 \%$ \\
\hline Total & 35 & 15 & 50 & $100 \%$ \\
\hline
\end{tabular}

The commonest age group was the $4^{\text {th }}, 5^{\text {th }}$ and $6^{\text {th }}$ decade. The average age is 52 . The lowest is 32 yrs and oldest 75 yrs.

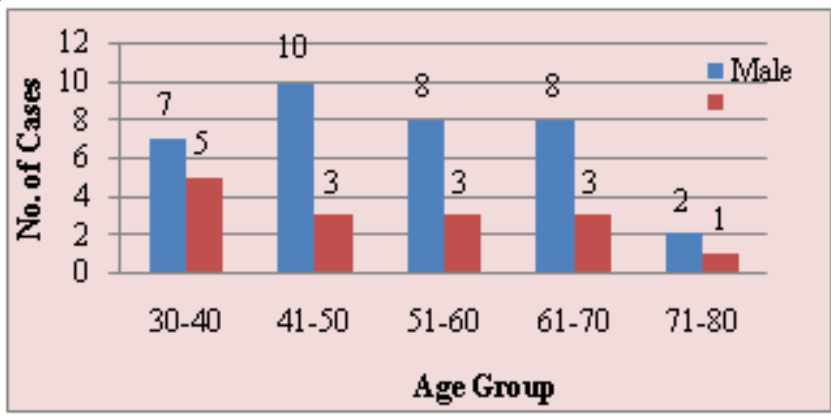

Site of lesion in advanced oral cavity cancer.

\begin{tabular}{|c|c|c|}
\hline Site & No. of Cases & Percentage \\
\hline Hard Palate & 1 & $2 \%$ \\
\hline Tongue & 23 & $46 \%$ \\
\hline Buccal mucosa & 15 & $30 \%$ \\
\hline Lip & 3 & $6 \%$ \\
\hline Alveolus & 7 & $14 \%$ \\
\hline Floor of mouth & 1 & $2 \%$ \\
\hline Total & 50 & $100 \%$ \\
\hline
\end{tabular}

In this study most commonest site is Tongue $46 \%$.

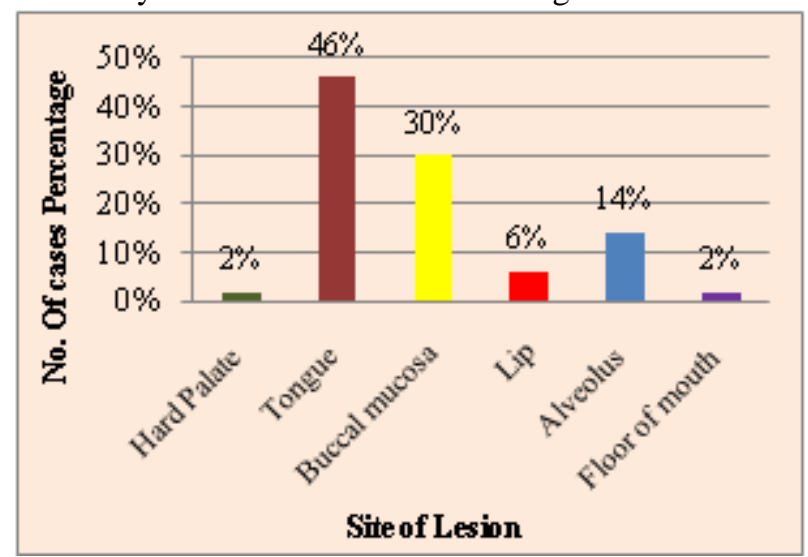

Tumor histology in advanced oral cavity cancer

\begin{tabular}{|c|c|c|}
\hline Histopathological Report & No. of Cases & Percentage \\
\hline Well diff. squa. Cell Ca. & 24 & $48 \%$ \\
\hline Moderately diff. squa. Cell Ca. & 22 & $44 \%$ \\
\hline Poorly diff. squa. Cell Ca. & 4 & $8 \%$ \\
\hline Total & 50 & $100 \%$ \\
\hline
\end{tabular}

Histopathologicaly well differentiated squamous cell carcinoma is commonest i.e 24 cases (48\%).

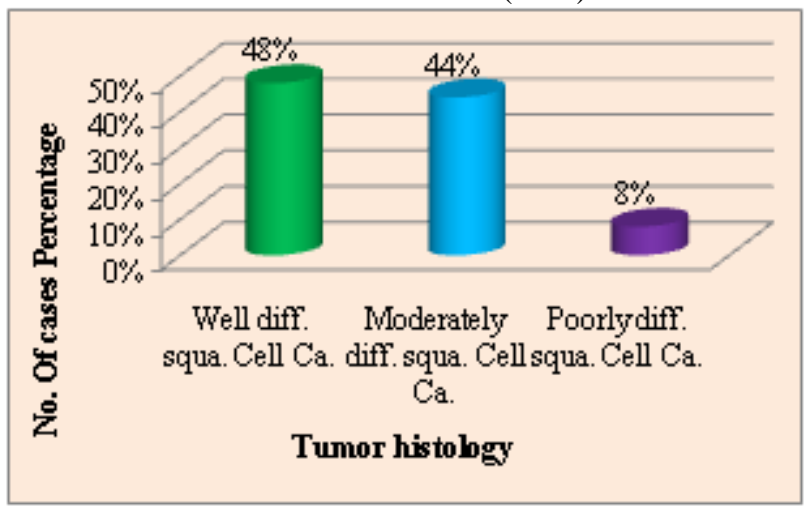

Response of Chemotherapy \& Radiotherapy with time < 1 Month

\begin{tabular}{|c|c|c|}
\hline Response & No. of Cases & Percentage \\
\hline Rapid & 26 & $52 \%$ \\
\hline Slow & 24 & $48 \%$ \\
\hline Total & 50 & $100 \%$ \\
\hline
\end{tabular}

In this study maximum patient responded chemotherapy rapidly i.e 52\%.

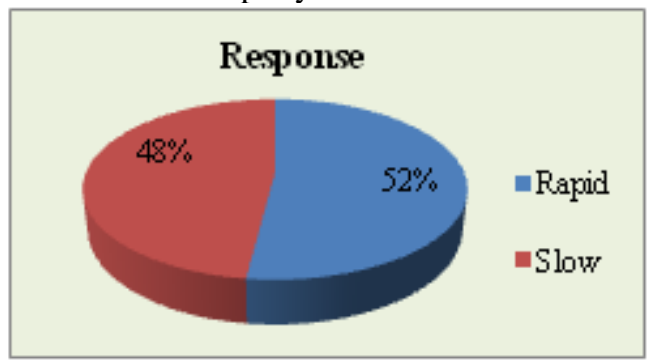

Correlation of remission with Stage of diseases.

\begin{tabular}{|c|c|c|c|}
\hline Remission & Stage III & Stage IV & Total \\
\hline C.R & 8 & 17 & 25 \\
\hline P.R & 2 & 19 & 21 \\
\hline C.R + P.R & 10 & 36 & 46 \\
\hline N.R & 0 & 4 & 4 \\
\hline Total & 10 & 40 & 50 \\
\hline
\end{tabular}

It can be seen in the above table response rate in the case of stage III in $100 \%$ \& stage IV $90 \%$

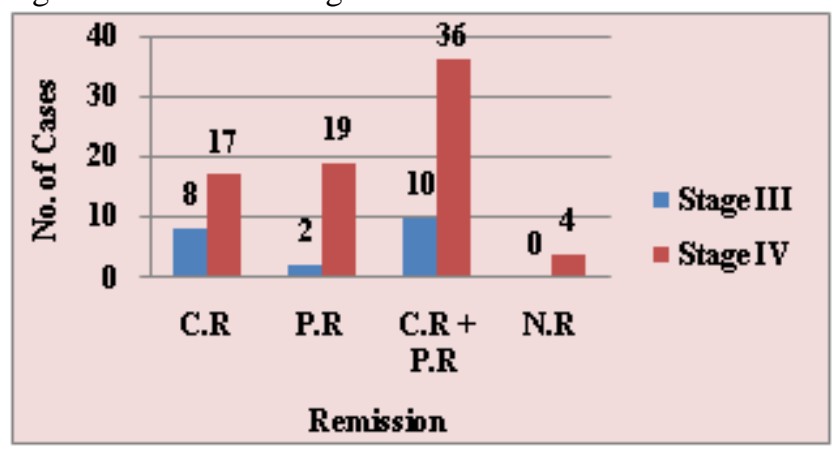




\section{International Journal of Science and Research (IJSR) \\ ISSN (Online): 2319-7064}

Index Copernicus Value (2013): 6.14 | Impact Factor (2015): 6.391

Correlation of remission depending on Histological Basis

\begin{tabular}{|c|c|c|c|}
\hline Remission & $\begin{array}{c}\text { Well } \\
\text { Differentiated }\end{array}$ & $\begin{array}{c}\text { Moderate } \\
\text { Differentiated }\end{array}$ & $\begin{array}{c}\text { Poor } \\
\text { Differentiated }\end{array}$ \\
\hline C.R & 22 & 3 & 0 \\
\hline P.R & 2 & 19 & 0 \\
\hline C.R + P.R & 24 & 22 & 0 \\
\hline N.R & 0 & 0 & 4 \\
\hline Total & 24 & 22 & 4 \\
\hline
\end{tabular}

Well Differentiated showing $100 \%$ of Response where as poorly differentiated shows $100 \%$ of no response.

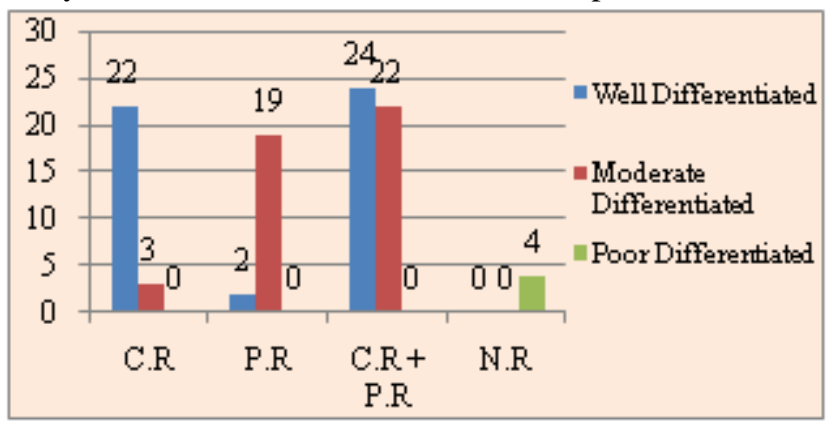

\section{Discussion}

The incidence of oral cavity cancer its distribution in the cavity, it's age incidence and sex ratio all very widely in different nations and races with difference in their nutritional status, their customs, their habit , their occupation and their awareness of general health and hygiene.

In our country the incidence of oral cavity cancer is very high as compared to western countries. Parmaster ${ }^{2}$ quotes an incidence of $45 \%$ of taotal cancers in india. Similarly Jussawalla quotes the incidence of $37.5 \%$ of all cancers. Local and regional control were better in the combined therapy than in group given radiotherapy alone. (95\% confidence interval). The estimated two year rate of local and regional control was $82 \%$ in the combined therapy as compared in radiotherapy group alone i.e. $72 \%$ as written in New England Journal of Medicine.

\section{Age Distributiion:}

In this series, the age range encountered is between 30 to 80 years. commenst were $4^{\text {th }}, 5^{\text {th }}$ decades. The average age is 52 yrs. Spiro quotes median age a 60 yrs. $^{3}$

\section{Sex Distribution:}

The sex wise incidence of cancers differs in different countries. In this series male to female ratio is 2.3:1. Mehta M.J. ${ }^{4-5}$ quotes male to female ratio 3:1.

\section{Socio Economis Status:}

In this series higher + middle and low socio economic, incidence of oral cancer in higher group and lower group is 4.5:1.

In my study tobacco with lime chewing is the most common habit in male and tobacco mishri in females.

Duration Of Symptoms In Advanced Oral Cancers:

In this study most of the case presented between 3-4 months.

\section{Site of the Tumors:}

In this study most common sites are tongue i.e. $46 \%$ and Buccal mucosa i.e. $30 \%$

\section{Histopathologically:}

Well differentiated squamous cell carcinoma is the commonest i.e. $48 \%$ followed by moderately differentiated SCC.

As in my study response in stage III was $100 \%$ and stage IV was $90 \%$. With only 4 patients out of fifty didn't respond to the treatment. A number of studies using combination of cisplatin and radiotherapy has been reported. Another randomized study conducted by University of Wisconsin, with the median follow up of 27 months, the Kaplan Meier shows in field tumor control of $89.1 \%$, regional control of $88.9 \%$ and overall survival of $86.9 \%$ at 2 years. With $95 \%$ of confidence interval.

Cisplatin is one of the favoured drugs because of its proved radiosesitizing effect and its different toxicities profile. As in randomized case study held in SKIMS College, Tumor response to treatment was recorded in all 45 patients. This included a complete response in 26 patients $(57.7 \%)$ and partial response in 14 (31.1\%) and in 5 (11.1\%) the disease remained stable. As in my study complete response was seen in 25 patients i.e. 50\% and partial response was seen in $42 \%$ i. e. 21 patients.

\section{Summary and Conclusion}

- This study included fifty patients of Stage III and Stage IV oral cavity cancer. Thirty five were male and fifteen were female.

- Their age varied between 30 to 80 years.

- Average age of disease was 52 years a commonest decade being the $5^{\text {th }}$ decade.

- It showed clear male preponderance, with male to female ration 2.3:1.

- Most of cases study in this series from rural area.

- Poor oral hygiene was observed in $72 \%$ majority of cases from lower socioeconomic class i.e. $82 \%$ and higher plus middle socioeconomic group is about $18 \%$.

- The most common habit in male is chewing tobacco and in female is tobacco mishri application.

- Most of the cases presented in the study between 3 to 4 months i.e. $54 \%$ cases.

- The commonest site of malignancy was tongue followed by buccal mucosa

- $48 \%$ cases were well-differentiated squamous cell carcinoma.

- 25 of them had complete remission and 21 patients had partial remission. 4 patients delivered the no response.

- Stage III tumours had better response than Stage IV tumours

- Toxicity observed was Nausea, vomiting, Leucopenia, Mucositis , Dysphagia.

- In conclusion combination of cisplatin - and radiotherapy given as once weekly and RT for 33 Fr 5 times a week found to be effective, economical and was better tolerated by the patients. 


\section{International Journal of Science and Research (IJSR) \\ ISSN (Online): 2319-7064}

Index Copernicus Value (2013): 6.14 | Impact Factor (2015): 6.391

\section{References}

[1] Jussawalla D. J. Yeole B. B. Natekar MV: Cancer morbidity and mortality greater Bombay, 1983. The Indian cancer society, Bombay 1986-87./1

[2] 2) Parmaster J.C.: The importance of uniform clinical classification and staging in carcinoma of upper aerodigestion tract, Indian Journal of surgery X, IX 1-6 : 1957.

[3] 3) Mehta M. J. A study of 81 cases of squanous cell ca, of buccal and gingval mucosa. Indian Jr. of surgery XXVI No. 4,306; 1964.

[4] 4) Jussawale D. J. Deshpande V.: A evaluation of cancer risk in tobacco chewers and smokers , An epidemological assessement cancer 28:244-252;1971.

[5] 5) Sabiston David C: Textbook of surgery, mouth cancer. Ch. 14. 14th edi. pg. 1320-1997. 